\title{
DIPROSOPIA relacionada à administração de progestágenos em felino doméstico neonato: relato de caso
}

\author{
Diprosopia related to the administration of progestogens \\ in domestic feline neonate: case report
}

\author{
João Luís T. Camassola1*; Tainá Ança Evaristo²; Beatriz Persici Maroneze ${ }^{3}$; Josiane Bonel ${ }^{4}$; \\ Eduardo de Bastos Santos Junior5; Fabrício Arigony Braga6; Patrícia Vives ${ }^{7}$
}

*Autor Correspondente: João Luís T. Camassola. Rua Dona Joaquina, 144, Kaiser, Caxias do Sul, Pelotas, RS, Brasil, CEP: 95096-381. E-mail: joaocamassola@hotmail.com

\begin{abstract}
Como citar: CAMASSOLA, João Luís T. et al. Diprosopia relacionada à administração de progestágenos em felino doméstico neonato: relato de caso. Revista de Educação Continuada em Medicina Veterinária e Zootecnia do CRMV-SP, São Paulo, v.18, n. 2, 2020. Doi 10.36440/recmvz.v18i1.37995

Cite as: CAMASSOLA, João Luís T. et al. Diprosopia related to the administration of progestogens in domestic feline neonate: case report. Journal of Continuing Education in Animal Science of CRMV-SP, São Paulo, v.18, n. 2, 2020. Doi 10.36440/recmvz.v18i1.37995
\end{abstract}

\section{Resumo}

A diprosopia é uma anomalia congênita na qual a região da cabeça e as estruturas faciais do animal se mostram duplicadas. Esse defeito congênito é mais comumente relatado em bovinos, mas também ocorre em felinos, caninos, caprinos, ratos e veados. 0 presente trabalho objetivou relatar um caso de diprosopia associada a fenda palatina bilateral em felino neonato. Uma gata não castrada foi atendida em uma clínica particular situada na cidade de Porto Alegre/RS, manifestando prostração e, segundo o tutor, havia administrado progestágenos anteriormente. Ao exame físico foi vista uma membrana similar a vesícula embrionária ao toque vaginal. A ecografia abdominal constatou gestação e sofrimento fetal, optandose pela cirurgia. Dos cinco neonatos, um estava morto, três vieram a óbito em torno de 24 horas após o procedimento e um destes apresentava alterações sugestivas de diprosopia associada a fenda palatina bilateral, e foi encaminhado para necropsia. Considerada uma má formação congênita pouco frequente em felinos, a diprosopia pode estar associada ao uso prévio de progestágenos ao período gestacional em gatas. Palavras-chave: Cirurgia. Anticoncepcional. Malformação congênita. Distocia.

\footnotetext{
1 Discente em Medicina Veterinária, Faculdade de Veterinária, Universidade Federal de Pelotas, Pelotas, RS, Brasil

2 Discente em Medicina Veterinária, Faculdade de Veterinária, Universidade Federal de Pelotas, Pelotas, RS, Brasil

3 Médica-veterinária, especialista em Clínica Cirúrgica de Animais de Companhia, Universidade Federal de Pelotas, Pelotas, RS, Brasil

4 Doutora, docente da Faculdade de Veterinária, Universidade Federal de Pelotas, Pelotas, RS, Brasil

5 Médico-veterinário, sócio-proprietário e cirurgião da Clínica Veterinária do Forte, Porto Alegre, RS, Brasil

6 Doutor, docente do Departamento de Clínicas Veterinárias, Universidade Federal de Pelotas, Pelotas, RS, Brasil

7 Doutora, técnica em Educação, Hospital Veterinário FAVET, Universidade Federal de Pelotas, RS, Brasil
} 


\section{Abstract}

Diprosopia is a congenital anomaly in which the head region and facial structures of the animal are doubled. This congenital defect is most commonly reported in bovines, but it also occurs in felines, canines, goats, rats and deer. The present study aimed to report a case of diprosopia associated with bilateral cleft palate in neonate feline. An uncastrated cat was treated at a private clinic in the city of Porto Alegre / RS, manifesting prostration, to the vaginal touch there was a membrane similar to the embryonic vesicle and, according to the tutor, had previously administered progestogens. Abdominal ultrasound found gestation and fetal distress, opting for surgery. Of the five newborn pups, one was dead, another three died about 24 hours after the procedure and one of them presented alterations suggestive of diprosopia associated with bilateral cleft palate, being referred for necropsy. Considered an infrequent congenital formation in felines, diprosopia may be associated with the previous use of progestogens to the gestational period in cats.

Keywords: Surgery. Contraceptive. Congenital malformation. Dystocia.

\section{Introdução}

As anomalias congênitas são definidas como anormalidades da estrutura ou função presentes ao nascimento que podem ser causados por fatores genéticos ou ambientais, ou ainda pela combinação de ambos (MIRANDA et al., 2013) o que na maioria das vezes culmina no óbito do indivíduo (RADOSTIS et al., 2007).

Entre os fatores com maior importância para a indução de malformações estão o uso de progestágenos abortivos, distúrbios nutricionais, agentes teratogênicos como radiação, traumas, uso de drogas como os benzimidazóis e cortisona, e a ingestão de plantas tóxicas durante o período gestacional (RADOSTIS et al., 2007).

A diprosopia é uma anomalia congênita na qual a região da cabeça e as estruturas faciais do animal se mostram duplicadas (POTTER \& CRAIG, 1975). Sua etiologia geralmente é indefinida, no entanto, pode estar associada a defeitos congênitos de células germinativas ou causada por influências ambientais que agem no desenvolvimento fetal (SCHULZE et al., 2006). Esta malformação congênita pode ocorrer em gatos domésticos, bovinos, caprinos, ovinos, ratos e veados (ZAITOUN, 1999), com maior incidência em bovinos frente às outras espécies (SILVA et al., 2010).

0 presente trabalho tem como objetivo relatar um caso de diprosopia associada à fenda palatina bilateral em um felino neonato.

\section{Relato de caso}

Uma gata fêmea não castrada, raça siamês e com cinco anos de idade, foi levada até uma clínica particular situada na cidade Porto Alegre/RS, para uma consulta, com a principal queixa de inapetência e prostração.

0 tutor relatou que periodicamente administrava acetato de medroxiprogesterona $(1 \mathrm{ml} /$ animal), porém a última aplicação havia sido feita havia dois anos e que após este período, a gata já havia parido filhotes hígidos e por parto normal.

No exame físico geral as alterações observadas foram mucosas hipocoradas, temperatura corpórea de $36,5^{\circ} \mathrm{C}$ e ao toque vaginal foi constatada a presença de uma membrana semelhante a vesícula embrionária. 
A paciente foi encaminhada para o setor de imagem e o ultrassom abdominal confirmou a gestação. Havia grande quantidade de líquido amniótico, os diâmetros dos crânios indicavam fetos com 52 dias e 180 batimentos cardíacos por minuto, revelando sofrimento fetal. Diante do quadro a paciente foi encaminhada para cesariana de emergência.

Foi realizado o preparo pré-cirúrgico de rotina e plano anestésico, utilizando os seguintes fármacos: medicação pré-anestésica com metadona ( $0,5 \mathrm{mg} / \mathrm{kg} / \mathrm{l} . \mathrm{M}$.), indução com propofol ( $5 \mathrm{mg} / \mathrm{kg} /$ E.V.) e manutenção via inalatória com isoflurano (1,5 CAM), associado ao bloqueio epidural, com lidocaína $(5 \mathrm{mg} / \mathrm{kg})$ e morfina $(0,1 \mathrm{mg} / \mathrm{kg})$. Durante a celiotomia optou-se pela ovariosalpingohisterectomia (OSH) modificada em bloco, devido ao quadro clínico da paciente e, também para inibir futuras concepções.

Imediatamente após a retirada dos ovários, cornos e corpo do útero, estes foram entregues aos volantes para a célere remoção dos neonatos. Foram constatados cinco filhotes, destes, um estava em óbito e quatro natimortos. Dentre estes, um portador malformação sugestiva de diprosopia associada a fenda palatina bilateral.

A paciente apresentou excelente recuperação no pós-operatório, teve alta clínica e ao retorno para remoção dos pontos apresentava-se completamente reestabelecida.

O corpo do natimorto portador da duplicação craniana foi conservado em formalina $10 \%$ e encaminhado para necropsia, concluindo que o indivíduo apresentava diprosopia.

A necropsia revelou um único corpo de um felino neonato contendo vísceras anatômicas até a região cervical, porém, apresentava duplicidade morfológica na extremidade oral e fenda palatina em ambas as cavidades orais. A duplicação ocorreu a partir da epiglote em direção oral e os crânios não estavam separados, embora houvesse dois encéfalos e um cerebelo (Figura 1).

Figura 1. Imagens da necropsia de um felino natimorto conservado em formalina $10 \%$, com $13,5 \mathrm{~cm}$ de comprimento portador de diprosopia, posicionado em decúbito ventral (A) e decúbito dorsal (B). Imagens detalhadas da duplicação craniofacial (diprosopu) em vista rostral (C) e evidenciando as fendas palatinas (D). Vista rostro-dorsal após a remoção da calota craniana para demonstrar a diencefalia (E). Decúbito dorsal com exteriorização das cavidades pélvica, abdominal, torácica e oral (F). Exposição cervical ventral, demonstrando apenas uma traqueia (G-seta). Retirada em bloco dos pulmões, esôfago e traqueia, evidenciando duas epiglotes no ponto onde ocorre a bifurcação oral (H-seta)
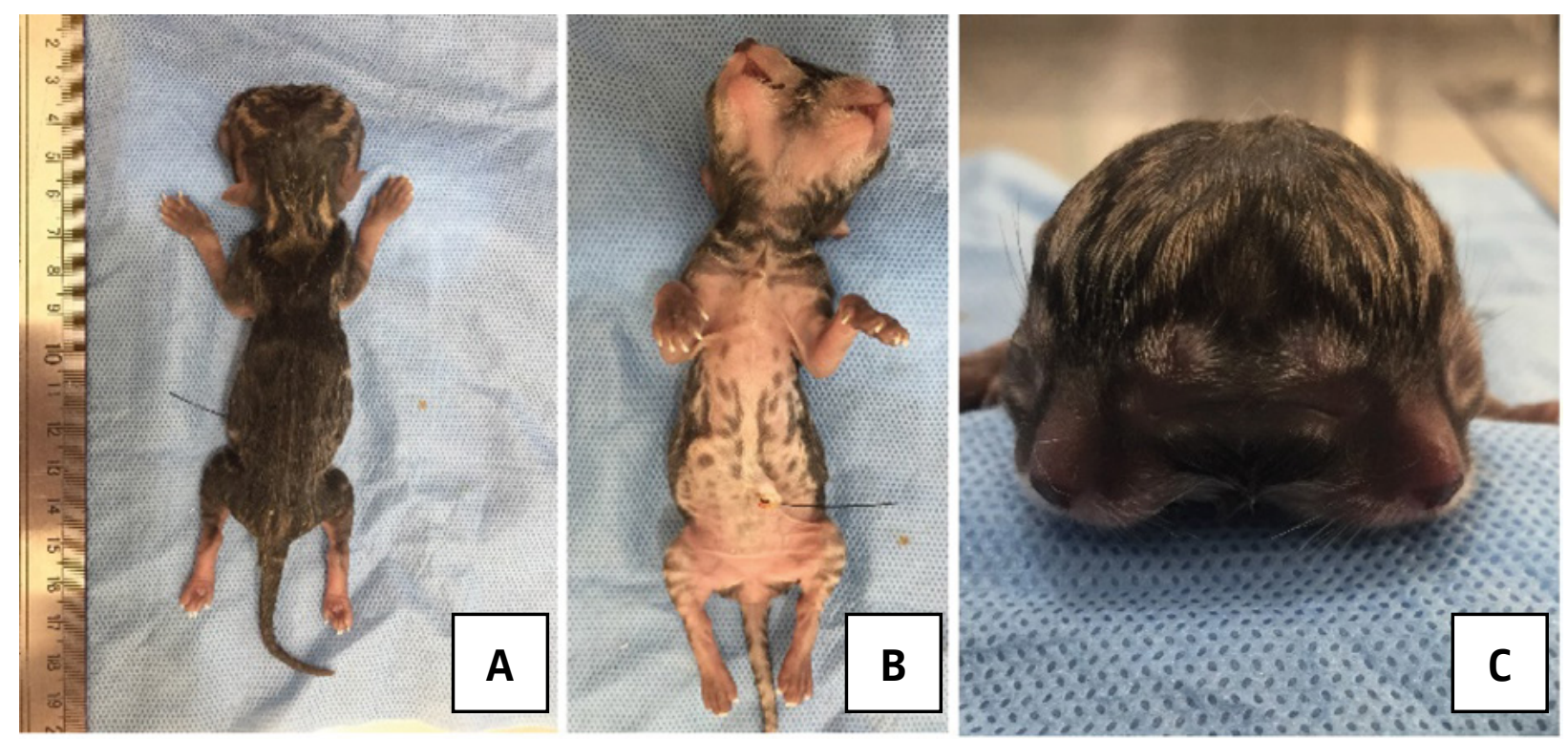

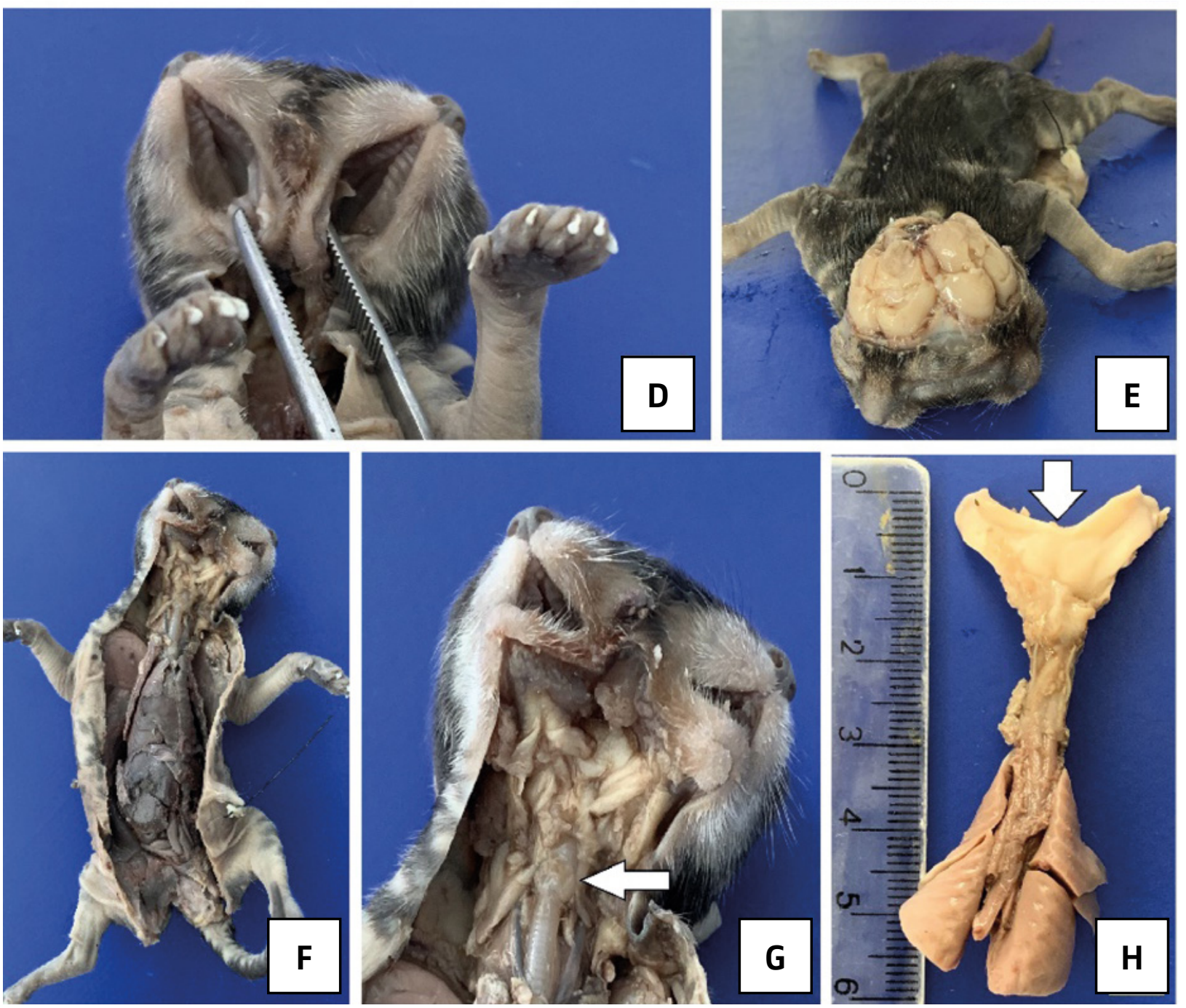

Fonte: Autor (2019).

\section{Discussão}

Malformações congênitas decorrem de falhas no desenvolvimento embrionário que resultam em anormalidades funcionais e/ou estruturais. A parapagia cefálica é classificada como diprosopia ou dicefalia (ROBERTS, 1986). A germinação de diprosopus se refere a um único corpo unido com mais de uma cabeça, mostrando aspecto de duplicações de estrutura craniofaciais com grande variabilidade em seu local de união e grau de compartilhamento de órgãos.

Em casos extremos de geminação de diprosopus são visualizadas duas faces com focinhos completos (ROBERTS, 1986), conforme a descrição no presente trabalho, entretanto, dicephalus refere-se a duas cabeças totalmente separadas, o que diverge neste relato.

A duplicação parcial da traqueia e do esôfago é a anormalidade mais relatada em neonatos bovinos diprosopus (ARTHUR, 1956). Neste relato, o felino apresentava diencefalia, porém os pulmões, traqueia e esôfago eram únicos, ocorrendo a bifurcação apenas após a passagem da epiglote, compreendo duas cavidades orais.

A duplicação craniofacial possui causas indefinidas e pode estar associada a defeitos congênitos de células germinativas, ou ainda influências medicamentosas e ambientais que intervêm no desenvolvimento normal do feto (CORNILLI; JACOBS; BREUGELMANS, 2006). Alterações em palato, como a fenda palatina relatada, estão associadas a causas nutricionais, mecânicas (compressões 
uterinas), hormonais ou tóxicas, sendo a causa mais comum o fator hereditário (ROBERTSON, 1996; NELSON, 1998).

O uso de contraceptivos em gatas é um método comum para evitar gestações, cios e mudanças comportamentais, porém frequentemente são administrados sem prescrição veterinária e sem finalidade terapêutica (ADAMS, 2003). No presente relato, esta fêmea recebeu aplicações prévias de acetato de medroxiprogesterona, contraceptivo subcutâneo com propriedades e ações antiestrogênicas e antigonadotropínicas, contraindicado durante as fases do ciclo estral, casos de falsa prenhez, cios irregulares ou alguma anormalidade no sistema reprodutor (ADAMS, 2003).

O uso de progestágenos no período gestacional eleva significativamente as alterações primárias, como maceração fetal pela contração prolongada da cérvix e também alterações secundárias como piometra, hiperplasia mamária, diabetes mellitus e malformações fetais (MONTANHA; CORRÊA; PARRA, 2012). 0 tutor não soube informar em qual fase estral a fêmea encontrava-se no momento da última aplicação de acetato de medroxiprogesterona, mas a ocorrência desta malformação fetal corrobora com os achados literários.

\section{Considerações finais}

A diprosopia é uma má formação congênita incompatível com a vida, pouco frequente em felinos e pode estar associada ao uso prévio de progestágeno no período gestacional em gatas.

\section{Referências}

ADAMS, H. R. Farmacologia e terapêutica em Veterinária. 8. ed. Guanabara Koogan: Rio de Janeiro, p. 224-248, 2003.

ARTHUR, G.H. Conjoined twins-the veterinary aspects. Vet. Rec. 68, p. 389-393, 1956.

CORNILLI, P.; JACOBS, C.; BREUGELMANS, S. Abnormal connections between the digestive tract and respiratory system is a frequent finding in dicephalices calves. Italy Journal Anatomy and Embryology, v. 111, p. 99, 2006.

MIRANDA, D. F. H. et al. Malformações congênitas em neonatos felinos: relato de caso. PUBVET, v. 7, p. 259-311, 2013.

MONTANHA, F. P.; CORRÊA, C. S. D. S.; PARRA, T. C. Maceração fetal em gata em decorrência do uso de contraceptivos: relato de caso. Revista Científica Eletrônica de Medicina Veterinária, v. 10, n. 19, p. 1-6, 2012.

NELSON, A. W. Sistema respiratório superior. In: SLATTER, D. Manual de cirurgia de pequenos animais. 2. ed. São Paulo: Manole, v. 1, p. 884-935, 1998.

POTTER, E.L.; CRAIG, J.M. Multiple pregnancies and conjoined twins. In: POTTER, E.L.; CRAIG, J.M. (Eds). Pathology of the Fetus and Infant. 3. ed. Chicago: Year Book Medical Publishers, p. 207-230, 1975.

RADOSTIS, O. M. et al. Veterinary medicine. 10. ed. London, UK: Saunders, p. 619-620, 2007. 
ROBERTS, S. J. Veterinary obstetrics and genital disease (Theriogenelogy). USA/New York: Woodstock, p. 981, 1986. Disponível em: https://doi.org/10.1016/0093-691X(86)90160-3. Acesso em: 01 set. 2020.

ROBERTSON, J. J. Palato. In: BOJRAB, M. J. Mecanismos da moléstia na cirurgia dos pequenos animais. São Paulo: Manole, p. 225-228, 1996.

SCHULZE, U. et al. Familial occurence of diprosopus in german holstein calves. Berliner und Münchener tierärztliche Wochenschrift, v. 119, p. 251-257, 2006.

SILVA, A. et al. Diprosopus em bezerro: relato de caso. Revista Científica Eletrônica de Medicina Veterinária, Garça/SP, 2010. Disponível em: http://faef.revista.inf.br/imagens arquivos/ arquivos destaque/IE41SIYrKdrAva5 2013-6-25-14-58-10.pdf. Acesso em: 15 ago. 2019.

ZAITOUN, A.A.M.; CHANG, J.; BOOKER, M. Diprosopus (partially duplicated head) associated with anencephaly: a case report. Pathology, Research and Practice, v.195, p.45-50, 1999. Disponível em: https://doi.org/10.1016/s0344-0338(99)80094-6. Acesso em: 24 ago. 2020. 